\title{
Spin Frustration in Some Magnetic Compounds
}

\author{
A. Szytula ${ }^{a, *}$, E. $\operatorname{GondeK}^{a}$, B. $\operatorname{PenC}^{a}$ \\ AND J. HERNANDEZ-VELASCO ${ }^{b}$ \\ ${ }^{a}$ M. Smoluchowski Institute of Physics, Jagiellonian University \\ Reymonta 4, 30-059 Kraków, Poland \\ ${ }^{b}$ BENSC, Hahn-Meitner Institute \\ Glienicker Str. 100, 14-109 Berlin-Wannsee, Germany
}

\begin{abstract}
In the paper the results of the powder neutron diffraction measurements of RAuIn ( $\mathrm{R}=\mathrm{Ce}$, Tb, Dy, and $\mathrm{Er})$ and $\mathrm{ZnFe}_{2} \mathrm{O}_{4}$ compounds at low temperatures are presented. For the RAuIn compounds, which crystallize in the hexagonal ZrNiAl-type structure, the rare-earth moments lie in the $a b$-plane and form a typical triangle non-collinear structure. $\mathrm{ZnFe}_{2} \mathrm{O}_{4}$ has the normal spinel structure in which the $\mathrm{Fe}^{3+}$ ions occupy the $\mathrm{B}$ sites, forming the corner-shared tetrahedra of the Fe spins. At low temperatures experimental data indicate the coexistence of the long-range and the short-range magnetic order. The obtained results suggest that the important factor which influences the magnetic ordering in these compounds is geometrical frustration of magnetic moments.
\end{abstract}

PACS numbers: 75.10.Nr, 75.25.+z, 75.50.Ee

\section{Introduction}

Geometrically frustrated systems are ubiquitous and they are interesting because their behaviour is difficult to predict as frustration can lead to macroscopic degeneracy and qualitatively new states of matter. Magnetic systems offer good examples in the form of spin lattice.

The fundamental unit of frustration is antiferromagnetically interacting three spins on a regular triangle. A regular tetrahedron composed of four triangles serves

*corresponding author; e-mail: szytula@if.uj.edu.pl 
as the unit of three-dimensional frustrated lattices. Geometrically frustrated lattices are formed by connecting their edges or corners. The triangular lattice and the face centered cubic lattice are edge-sharing lattices of triangles and tetrahedra, respectively. Corner sharing of triangles yields the Kagomé lattice while a tetrahedron forms a lattice presented in Fig. 1. In the last type structure there crystallize a number of magnetic materials belonging to the different classes of crystal symmetry such as normal spinel, pyrochlores $\left(\mathrm{Y}_{2} \mathrm{Mo}_{2} \mathrm{O}_{7}\right)$, and $C 15$ Laves phase intermetallic compounds such as $\mathrm{Y}(\mathrm{Sc}) \mathrm{Mn}_{2}$. In all these systems the magnetic ions form corner-shared tetrahedrons as depicted in Fig. 1. In these systems the three-dimensional geometrical frustration exists which caused their unusual ground state, e.g., spin glass [1-3], spin ice [4], spin liquid and others [5, 6].

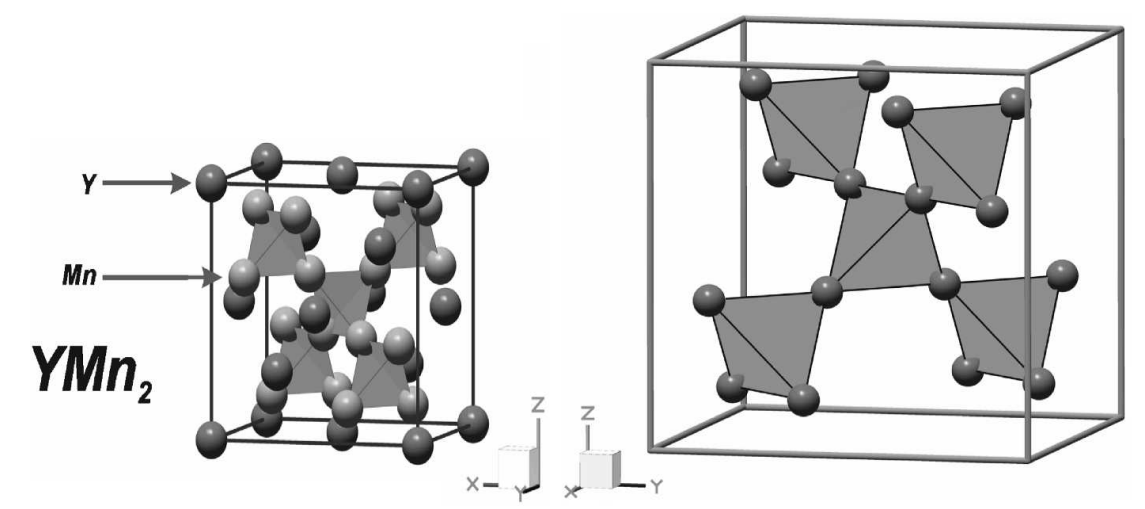

Fig. 1. The cubic corner-sharing tetrahedron lattice and the crystal structure of $\mathrm{YMn}_{2}$.

In this paper the results of experimental investigations of the two groups of compounds: RAuIn intermetallic compounds and spinel zinc ferrite $\mathrm{ZnFe}_{2} \mathrm{O}_{4}$ are discussed.

\section{Results and discussion}

\subsection{RAuIn compounds}

RAuIn compounds crystallize in the hexagonal ZrNiAl-type structure (space group $P \overline{6} 2 m$ ) [7]. The atoms occupy the following positions in the unit cell:

$\mathrm{R}$ atoms the $3 \mathrm{~g}$ site: $x_{\mathrm{R}}, 0,1 / 2 ; 0, x_{\mathrm{R}}, 1 / 2 ; \bar{x}_{\mathrm{R}}, \bar{x}_{\mathrm{R}}, 1 / 2$ with $x_{\mathrm{R}}=0.509 ;$

$\mathrm{Au}$ atoms the $1 \mathrm{~b}$ site: $0,0,1 / 2$ and $2 \mathrm{c}$ site: $1 / 3,2 / 3,0 ; 2 / 3,1 / 3,0$;

In atoms the $3 f$ site: $x_{\text {In }}, 0,0 ; 0, x_{\text {In }}, 0 ; \bar{x}_{\text {In }}, \bar{x}_{\text {In }}, 0$ with $x_{\text {In }}=0.265$.

The distribution of the rare-earth atoms in the basal plane is similar to the Kagomé lattice (Fig. 2). Magnetic measurements show that these compounds 

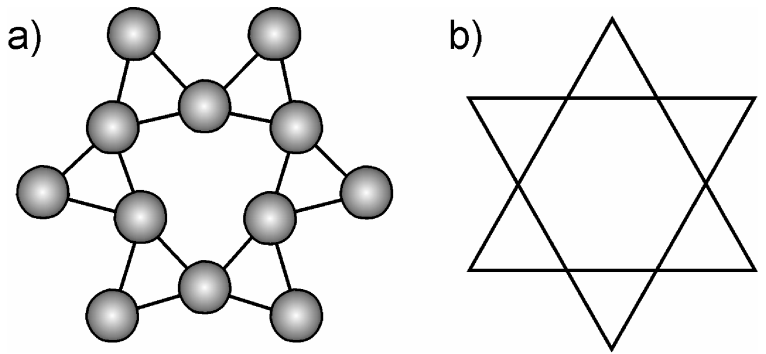

Fig. 2. (a) Projection of the hexagonal ZrNiAl-type structure on the basal plane. Only rare-earth atoms are presented, (b) Kagomé lattice.

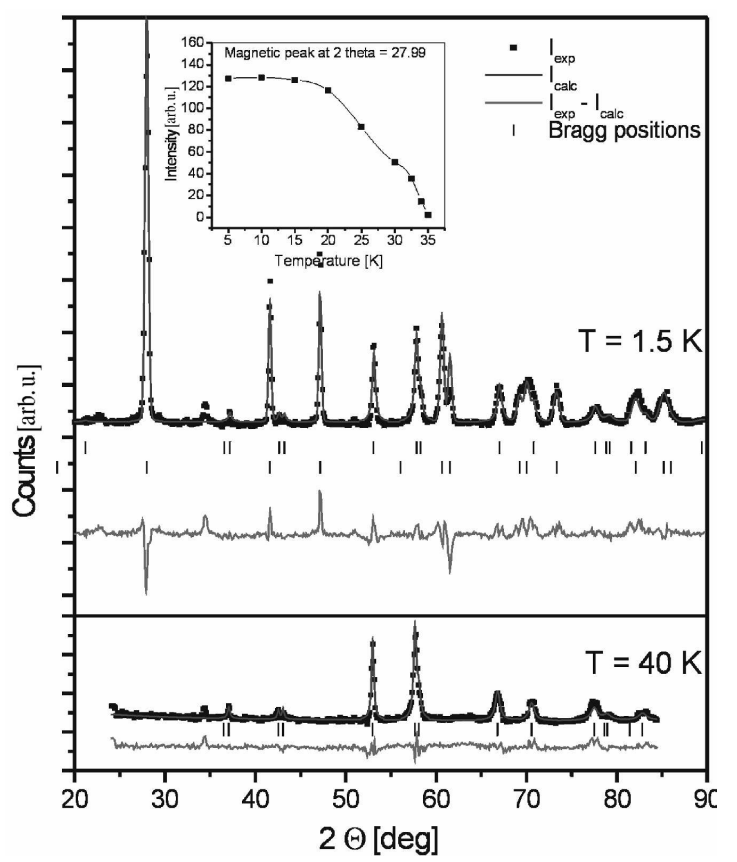

Fig. 3. Neutron diffraction patterns of TbAuIn at 1.5 and $40 \mathrm{~K}$. The squares represent experimental points; the solid lines are the calculated profiles for crystal and magnetic structure model and the difference between observed and calculated intensities at the bottom of each diagram. The vertical bars indicate the Bragg peaks of nuclear and magnetic origin. The inset shows the temperature dependence of the magnetic peak intensity.

are antiferromagnets at low temperatures. Our neutron diffraction measurements confirm those and permit determination of the magnetic structure of these compounds. Typical neutron diffraction patterns of TbAuIn measured at 1.5 and $40 \mathrm{~K}$ are shown in Fig. 3. The other investigated compounds have similar diffraction patterns at low temperatures. 


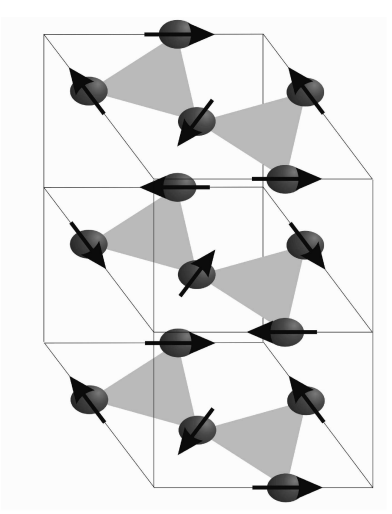

Fig. 4. Typical magnetic structure observed in RAuIn compounds.

The analysis of these patterns indicates that the rare-earth magnetic moments form non-collinear structure in the $a b$-plane in CeAuIn below $T_{\mathrm{N}}=6 \mathrm{~K}$, TbAuIn below $T_{\mathrm{N}}=35 \mathrm{~K}$, DyAuIn below $T_{\mathrm{N}}=11 \mathrm{~K}$, and ErAuIn below $T_{\mathrm{N}}=3 \mathrm{~K}$. Magnetic cell is doubled along the $c$-axis due to the propagation vector $\boldsymbol{k}=$ (0 $01 / 2)$. The rare-earth moments in the $a b$-plane form a typical triangle structure (see Fig. 4). The angle between the nearest neighbouring rare-earth moments is equal to $120^{\circ}$.

From the above results the following conclusions can be drawn:

- due to large $\mathrm{R}-\mathrm{R}$ interatomic distances of about $4 \AA$ the Ruderman-KittelKasuya-Yoshida (RKKY) model is believed to be suitable for magnetic interaction description,

- geometrical frustration of antiferromagnetic interaction influences the stability of magnetic structures.

\section{2. $\mathrm{ZnFe}_{2} \mathrm{O}_{4}$ compound}

$\mathrm{ZnFe}_{2} \mathrm{O}_{4}$ is a normal spinel. The cubic spinel structure of $(\mathrm{A})\left[\mathrm{B}_{2}\right] \mathrm{O}_{4}$ compounds is characterized by the cations $\mathrm{A}$ and $\mathrm{B}$ which are tetrahedrally () and octahedrally [] coordinated by the oxygen atoms. In $\mathrm{ZnFe}_{2} \mathrm{O}_{4}$ the $\mathrm{Fe}^{3+}$ ions occupy the $\mathrm{B}$ site, thus forming the corner-shared tetrahedral of spins on the Fe sites. This compound has a transition to an antiferromagnetic state at $10 \mathrm{~K}$. However, estimates based on magnetic susceptibility give the Curie-Weiss temperature of $120 \mathrm{~K}$ [8]. The magnetic properties were the subject of extensive studies by different authors [9-16]. The results presented by the individual authors are in contradiction. The partial results, particularly by the Mössbauer spectroscopy, suggest existence of a long-range order while those from neutron diffraction suggest a short-range order or a coexistence of a short-range (SRO) and a long-range (LRO) order below the Néel temperature. The new temperature dependence of magnetic susceptibility measured in broad temperature range up to $700 \mathrm{~K}$ suggests: 
- the field cooling and zero field cooling curves trace almost the same path over the whole temperature range. This indicates that the $\mathrm{ZnFe}_{2} \mathrm{O}_{4}$ is unlike a spin glass system,

- deviation of the reciprocal magnetic susceptibility from the Curie-Weiss law below $280 \mathrm{~K}$ together with the high value of the paramagnetic Curie temperature (equal to $120 \mathrm{~K}$, as estimated from the high-temperature region) indicate that the ferromagnetic spin correlation dominates at high temperatures [8].

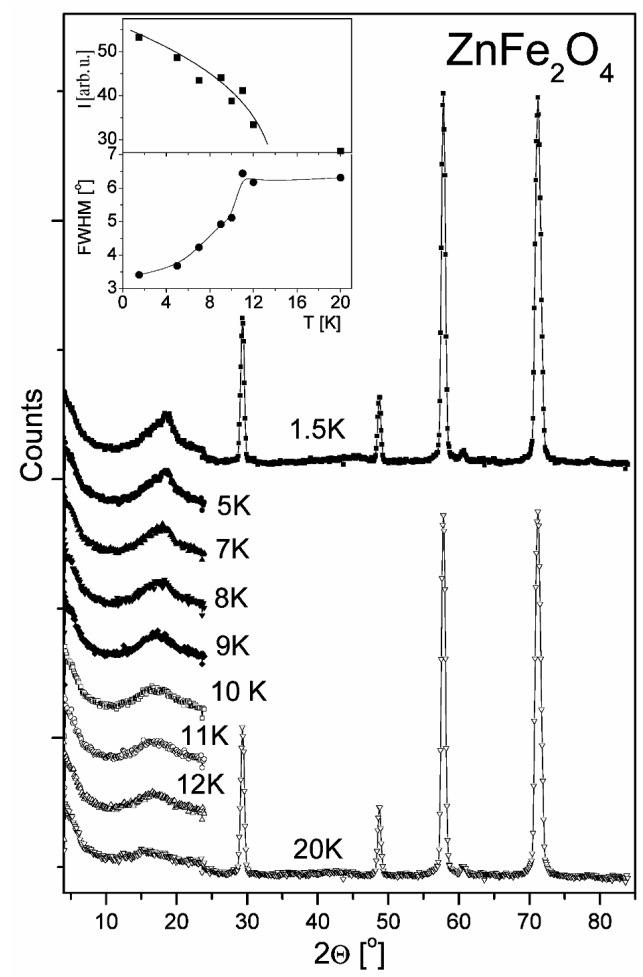

Fig. 5. Neutron diffraction patterns of $\mathrm{ZnFe}_{2} \mathrm{O}_{4}$ at different temperatures between 1.5 and $20 \mathrm{~K}$. The inset shows the temperature dependence of the intensity and the full-width at half-height of the broad hump.

The neutron diffraction patterns recorded at different temperatures between 1.5 and $20 \mathrm{~K}$ are shown in Fig. 5. The pattern measured at $20 \mathrm{~K}$ confirms the cubic spinel-type structure. The space group of the spinel-type structure is $F d 3 m$ (No. 227). The atoms occupy the following positions:

Zn atoms the 8 a site: $0,0,0$; 
Fe atoms the $16 \mathrm{~d}$ site: $5 / 8,5 / 8,5 / 8 ; 5 / 8,7 / 8,7 / 8$;

$\mathrm{O}$ atoms the $32 \mathrm{e}$ site: $u, u, u ; 1 / 4-u, 1 / 4-u, 1 / 4-u$.

Analysis of the pattern confirms that $\mathrm{ZnFe}_{2} \mathrm{O}_{4}$ is a normal spinel. The refined values of the lattice parameter $a$, the oxygen parameter $u$ at 1.5 and $20 \mathrm{~K}$ are listed in Table I. These parameters do not change with the change of temperature.

\section{TABLE I}

Crystal structure parameters of $\mathrm{ZnFe}_{2} \mathrm{O}_{4}$.

\begin{tabular}{l|c|c}
\hline \hline & $20 \mathrm{~K}$ & $15 \mathrm{~K}$ \\
\hline$a[\AA]$ & $8.4472(15)$ & $8.4467(15)$ \\
$u$ & $0.3844(4)$ & $0.3837(4)$ \\
$U$ & $5.08(34)$ & $4.66(34)$ \\
$V$ & $-4.01(33)$ & $-3.61(33)$ \\
$W$ & $1.05(7)$ & $-0.95(7)$ \\
$R_{\text {Brags }}[\%]$ & 0.96 & 0.39 \\
$R_{\text {prof }}$ & 1.47 & 0.44 \\
\hline
\end{tabular}

$a$ - the lattice parameter, $u$ - the positional parameter of the oxygen; $U, V, W$ - parameters which described the full-width at half-maximum of the Bragg reflection; $H_{k}^{2}=U \tan ^{2} \Theta+V \tan \Theta+W$

Inspection of the neutron diffraction patterns at $1.5 \mathrm{~K}$ shows the coexistence of the small intensity antiferromagnetic $101 / 2$ peak and a broad hump. The antiferromagnetic peak disappears at 7 while the intensity of the broad hump quickly dwindles and the hump widens up to $13 \mathrm{~K}$. At $20 \mathrm{~K}$ this broad hump of the small intensity still exists. The observed results are in agreement with the model proposed in Ref. [15], in which small antiferromagnetic clusters coexist with the regions of short-range order. This phenomena and existence of the short-range order are not fully understood yet.

The data in Ref. [11] indicate a strong influence of thermal treatment on the magnetic properties of $\mathrm{ZnFe}_{2} \mathrm{O}_{4}$. The slowly cooled sample is an antiferromagnet with the LRO order below $10 \mathrm{~K}$ and SRO up to $80 \mathrm{~K}$. In the quenched sample only the SRO is observed below $35 \mathrm{~K}$.

Basing on the distribution of the $\mathrm{Fe}^{3+}$ ions in a normal spinel the nearest neighbour antiferromagnetic-type interaction is certainly inadequate to describe the ordering process in $\mathrm{ZnFe}_{2} \mathrm{O}_{4}$. The possible further interactions up to third neighbours are listed in Table II. It should be noticed that there are two different exchange paths to connect the third neighbours, so that there is a total of four parameters: $J_{1}, J_{2}, J_{3}$, and $J_{3}^{\prime}$.

The analysis of the neutron diffraction diffuse intensity [16] indicates that the nearest-neighbour interaction is ferromagnetic which is consistent with the 
TABLE II

List of the $n$-th nearest-neighbour $\mathrm{Fe}^{3+}$ pairs up to $n=4$ and the possible exchange paths via the oxygen anion 0 and the Aor the B-site metal. There are two different exchange paths for the third neighbours.

\begin{tabular}{l|c|c|c|c}
\hline \hline & $d$ & $z$ & Exchange path & $J$ \\
\hline nearest neighbour & $\sqrt{2}$ & 6 & B-B, B-O-B & $J_{1}$ \\
second-nearest neighbour & $\sqrt{6}$ & 12 & B-O-A-O-B & $J_{2}$ \\
third-nearest neighbour & $\sqrt{8}$ & 6 & B-O-B-O-B & $J_{3}$ \\
& & 6 & B-O-A-O-B & $J_{3}^{\prime}$ \\
fourth-nearest neighbour & $\sqrt{10}$ & 12 & B-O-A-O-B & $J_{4}$ \\
\hline
\end{tabular}

$d$ - the distance between the Fe atoms in $\frac{a}{4} ; z$ - the number of the Fe neighbour atoms

high-temperature magnetic data [8] while the third-neighbour interaction is antiferromagnetic.

Different analysis of the magnetic ordering in $\mathrm{ZnFe}_{2} \mathrm{O}_{4}$ could be done basing on the model proposed to explaining the magnetic ordering in pyrochlore materials $\mathrm{R}_{2} \mathrm{Ti}_{2} \mathrm{O}_{7}$ ( $\mathrm{R}$ is a rare-earth metal). This model, based on the concept of macroscopically degenerated ground state, was first introduced by Pauling [17] to describe the proton disorder in ice (see Fig. 6).
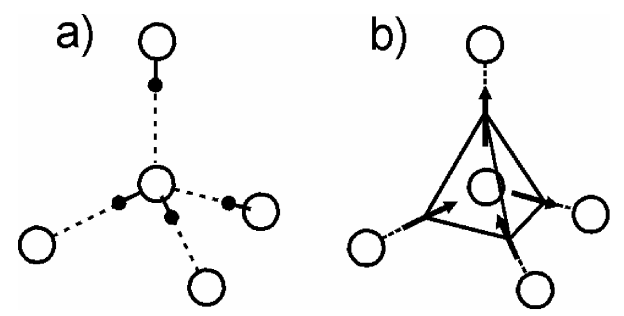

Fig. 6. (a) Local proton arrangement in water ice, showing oxide (large white circles) and hydrogen ions (small black circles); (b) the same as in (a) but now the positions of the protons are represented by the displacement vector (arrows) located at the midpoints of the oxide-oxide lines of contact.

The calculation of the frustrated Ising-type spin model in the tetrahedron sublattice is presented in Ref. [18]. The authors have calculated stability of magnetic structures considering the ferromagnetic or antiferromagnetic interactions and uniaxial or $\langle 111\rangle$ direction anisotropy. Stability of a magnetic structure is obtained for the uniaxial ferromagnet and the $\langle 111\rangle$ antiferromagnetic ordering (see Fig. 7).

Including a long-range interaction it is possible to obtain the ground state being a long-order (see Fig. 8a,b) [19] or not ordered system (Fig. 8c) [20]. 
a)

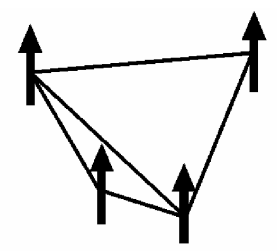

b)

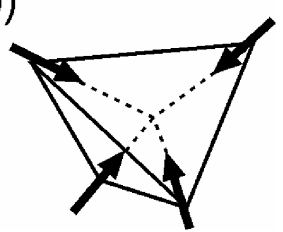

Fig. 7. The ground state of the single tetrahedron of spins for (a) ferromagnetic order with the uniaxial anisotropy and (b) antiferromagnetic order with the $\langle 111\rangle$ Ising anisotropy.

a)

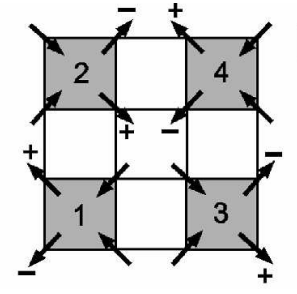

b)

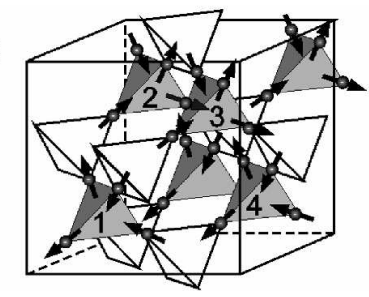

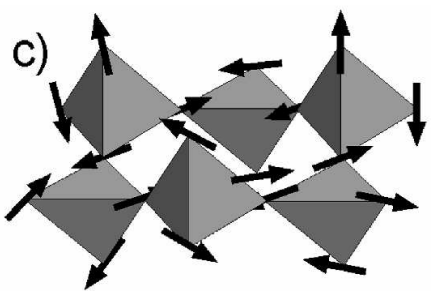

Fig. 8. (a, b) Predicted long-range order state in spin ice projected down the $z$-axis (a) and three-dimensional order; (c) not ordered state caused by spin fluctuations.

\section{Conclusions}

Presented in this paper results of the neutron diffraction data concerning the magnetic structures of RAuIn $(\mathrm{R}=\mathrm{Ce}, \mathrm{Tb}$, Dy, and $\mathrm{Er})$ and $\mathrm{ZnFe}_{2} \mathrm{O}_{4}$ indicate a strong influence of the crystal structure on the magnetic ordering. In the case of the RAuIn compounds geometrical frustration of the magnetic order is clearly visible.

The magnetic properties of $\mathrm{ZnFe}_{2} \mathrm{O}_{4}$ at low temperatures are not explained. From our data and the data of different authors it is not possible to determine the factors which are responsible for the different magnetic order at low temperatures. In this paper the problem of the magnetic order is discussed basing on the model proposed for the $\mathrm{R}_{2} \mathrm{Ti}_{2} \mathrm{O}_{7}$ compounds with the pyrochlore lattice. This model gives the spin ice order. Similar order of the Fe site is observed in the $\mathrm{CeFe}_{2}$ Laves phase [21].

\section{References}

[1] M.J.P. Gingras, C.V. Stager, N.P. Rain, B.D. Gaulin, J.E. Greedau, Phys. Rev. Lett. 78, 947 (1997).

[2] J.S. Gardner, B.D. Gaulin, S.-H. Lee, C. Broholm, N.P. Raju, J.E. Greedan, Phys. Rev. Lett. 83, 211 (1999).

[3] Amit Keren, J.S. Gardner, Phys. Rev. Lett. 87, 177202 (2001).

[4] H. Kadowaki, Y. Ishii, K. Matsuhira, Y. Hinatsu, Phys. Rev. B 65, 144421 (2002). 
[5] R. Ballou, E. Leliévre-Berna, Phys. Rev. Lett. 76, 2125 (1996).

[6] S.-H. Lee, C. Broholm, Y. Ueda, J.J. Rush, Phys. Rev. Lett. 86, 5554 (2001).

[7] Ł. Gondek, A. Szytuła, S. Baran, J. Hernandez-Velasco, J. Magn. Magn. Mater. 272-276, e443 (2004).

[8] K. Kamazawa, Y. Tsunoda, H. Kadowaki, K. Kohn, Phys. Rev. B 68, 024412 (2003).

[9] J.M. Hastings, L.M. Corlies, Phys. Rev. 10, 1463 (1956).

[10] U. Köning, E.F. Bertaut, Y. Gros, M. Mitrikov, G. Chol, Solid State Commun. 8, 759 (1970).

[11] W. Schäfer, W. Kockelmann, A. Kirfel, W. Potzel, F.J. Bughart, G.M. Kalvius, A. Martin, W.A. Kaczmarek, S.J. Campbell, Mater. Sci. Forum 321-324, 802 (2000).

[12] S. Ligenza, Phys. Status Solidi B 75, 315 (1976).

[13] W. Schiessl, W. Potzel, H. Karzel, M. Steiner, G.M. Kalvius, A. Martin, M.K. Krauze, I. Halevey, J. Gal, W. Schäfer, G. Will, M. Hillberg, R. Wäppling, Phys. Rev. B 53, 9143 (1996).

[14] B. Boucker, R. Buhl, M. Perrin, Phys. Status Solidi 40, 171 (1970).

[15] K. Kamazawa, Y. Tsunada, K. Kohn, J. Phys. Chem. Solids 60, 1261 (1999).

[16] Y. Yamada, K. Kamazawa, Y. Tsunoda, Phys. Rev. B 66, 064401 (2002).

[17] L. Pauling, The Nature of the Chemical Bond and the Structure of Molecules and Crystals: An Introduction to Modern Structural Chemistry, Cornell University Press, Ithaca 1960.

[18] S.T. Bramwell, M.J. Harris, J. Phys., Condens. Matter 10, L215 (1998).

[19] S.T. Bramwell, M.J.P. Gingras, Science 294, 1495 (2001).

[20] S.-H. Lee, C. Broholm, W. Ratcliff, G. Gasperovic, Q. Huang, T.H. Kim, S.-W. Cheong, Nature 418, 856 (2002).

[21] L. Paolasini, B. Ouladdief, N. Bernhoeft, J.-P. Sanchez, P. Vulliet, G.H. Lander, P. Canfield, Annual report of the Institute of Laue-Langevin, Grenoble 2002, p. 20. 